\title{
Amygdala Reactivity to Emotional Faces in the Prediction of General and Medication-Specific Responses to Antidepressant Treatment in the Randomized iSPOT-D Trial
}

\author{
Leanne M Williams ${ }^{*, 1,2,3,7}$, Mayuresh S Korgaonkar ${ }^{3,4,7}$, Yun C Song ${ }^{3}$, Rebecca Paton ${ }^{3}$, Sarah Eagles ${ }^{3}$, \\ Andrea Goldstein-Piekarski ${ }^{1,2}$, Stuart M Grieve ${ }^{3,4,5}$, Anthony WF Harris ${ }^{3,4}$, Tim Usherwood ${ }^{6,7,8}$ and \\ Amit Etkin ${ }^{1,2,8}$
}

'Department of Psychiatry and Behavioral Sciences, Stanford University, Stanford, CA, USA; ${ }^{2}$ Sierra-Pacific Mental Illness Research, Education, and Clinical Center (MIRECC) Veterans Affairs Palo Alto Health Care System, Palo Alto, CA, USA; ${ }^{3}$ The Brain Dynamics Center, Sydney Medical School, University of Sydney and Westmead Millennium Institute, Sydney, NSW. Australia; ${ }^{4}$ Discipline of Psychiatry, Sydney Medical School, University of Sydney, Westmead Hospital, Sydney, NSW, Australia; ${ }^{5}$ Sydney Translational Imaging Laboratory, Sydney Medical School, Sydney, NSW, Australia;

${ }^{6}$ Department of General Practice, Sydney Medical School Westmead, University of Sydney, Sydney, NSW, Australia

\begin{abstract}
Although the cost of poor treatment outcomes of depression is staggering, we do not yet have clinically useful methods for selecting the most effective antidepressant for each depressed person. Emotional brain activation is altered in major depressive disorder (MDD) and implicated in treatment response. Identifying which aspects of emotional brain activation are predictive of general and specific responses to antidepressants may help clinicians and patients when making treatment decisions. We examined whether amygdala activation probed by emotion stimuli is a general or differential predictor of response to three commonly prescribed antidepressants, using functional magnetic resonance imaging ( $\mathrm{fMRI}$ ). A test-retest design was used to assess patients with MDD in an academic setting as part of the International Study to Predict Optimized Treatment in Depression. A total of 80 MDD outpatients were scanned prior to treatment and 8 weeks after randomization to the selective serotonin reuptake inhibitors escitalopram and sertraline and the serotonin-norepinephrine reuptake inhibitor, venlafaxine-extended release (XR). A total of 34 matched controls were scanned at the same timepoints. We quantified the blood oxygen level-dependent signal of the amygdala during subliminal and supraliminal viewing of facial expressions of emotion. Response to treatment was defined by $\geqslant 50 \%$ symptom improvement on the 17-item Hamilton Depression Rating Scale. Pre-treatment amygdala hypo-reactivity to subliminal happy and threat was a general predictor of treatment response, regardless of medication type (Cohen's $d$ effect size 0.63 to 0.77 ; classification accuracy, $75 \%$ ). Responders showed hypo-reactivity compared to controls at baseline, and an increase toward 'normalization' post-treatment. Pre-treatment amygdala reactivity to subliminal sadness was a differential moderator of non-response to venlafaxine-XR (Cohen's d effect size I.5; classification accuracy, 81\%). Non-responders to venlafaxine-XR showed pre-treatment hyperreactivity, which progressed to hypo-reactivity rather than normalization post-treatment, and hypo-reactivity post-treatment was abnormal compared to controls. Impaired amygdala activation has not previously been highlighted in the general vs differential prediction of antidepressant outcomes. Amygdala hypo-reactivity to emotions signaling reward and threat predicts the general capacity to respond to antidepressants. Amygdala hyper-reactivity to sad emotion is involved in a specific non-response to a serotonin-norepinephrine reuptake inhibitor. The findings suggest amygdala probes may help inform the personal selection of antidepressant treatments.

Neuropsychopharmacology (20I5) 40, 2398-2408; doi:I0.1038/npp.20I5.89; published online 29 April 2015
\end{abstract}

\section{INTRODUCTION}

Major depressive disorder (MDD) is the leading cause of disability and has an economic impact in the United States of $\$ 42$ billion per year (World Health Organization, 2012).

*Correspondence: Professor LM Williams, Department of Psychiatry and Behavioral Sciences, Stanford University, 40I Quarry Road, Stanford, CA 94305, USA, Tel: +650 723 3579, Fax: +650 723 4655, E-mail: leawilliams@stanford.edu

${ }^{7}$ Equal first authors.

${ }^{8}$ Equal senior authors.

Received 27 August 2014; revised 3 March 2015; accepted 8 March 2015; accepted article preview online 3I March 2015
Typically, only about $50 \%$ of MDD patients respond to antidepressant treatment and even fewer achieve remission. There are currently no clinically useful means to predict response to antidepressant medications (Rush et al, 2008). Improved methods for objectively predicting response to medication could therefore have an important clinical impact. In this study, we focus on emotion processing, as this domain has been found to be perturbed in MDD and predict treatment outcome. We focus on the amygdala, as this region is implicated in abnormal emotion processing in MDD and its treatment (Hamilton et al, 2012; Stuhrmann et al, 2011; Whalen et al, 2002) and is a primary node of emotional brain circuits (Williams and Gordon, 2007). 
A robust way to probe amygdala activation is through the use of facial expressions of emotion (see the study by Williams and Gordon (2007) and Vuilleumier and Pourtois (2007) for a review). Facial expressions can be also used to define abnormalities in the processing of specific emotions, which may differ with respect to valence and arousal. One way to divide these emotional expressions is based on their communication of potential threat (fear, anger), loss (sad) and reward (happy). Using functional magnetic resonance imaging (fMRI), hyper-activation of the amygdala has been observed in MDD compared with healthy controls in response to threat-related expressions of anger and fear during recognition and matching tasks (Peluso et al, 2009; Yang et al, 2010) and during subliminal processing of these expressions when the patient is not directing their attention to the expressions in an effortful way (Sheline et al, 2001). Hyper-activation of the amygdala has also been observed during the supraliminal and subliminal processing of facial expressions of sadness (Surguladze et al, 2005; Fu et al, 2004; Victor et al, 2010; Arnone et al, 2012). The advantage of subliminal conditions is that they help to isolate the automatic processes that underpin amygdala activation from more elaborative supraliminal processes (Williams et al, 2006; Costafreda et al, 2008). Yet other studies have observed no differences between MDD patients and controls in amygdala reactivity for sad expressions (Almeida et al, 2010; Townsend et al, 2010). For positive expressions, the opposite finding of reduced amygdala activation in MDD has been reported (Stuhrmann et al, 2012; Suslow et al, 2010).

Previous treatment studies using emotion probes have focused on the effects of treatment on amygdala activation. Amygdala hyper-activation in MDD for the processing of facial expressions of sadness has been found to attenuate following treatment with the selective serotonin reuptake inhibitors (SSRIs) citalopram (Arnone et al, 2012), fluoxetine (Fu et al, 2004) and (in currently depressed patients) sertraline (Victor et al, 2010). For threat-related expressions, amygdala hyper-activity in MDD has also been found to attenuate in responders to escitalopram and paroxetine (Ruhé et al, 2012; Godlewska et al, 2012). There is also evidence that in healthy individuals, the acute administration of SSRIs reduces amygdala hyperactivation to threat-related faces (Harmer et al, 2006; Murphy et al, 2009), and corresponding effects have been observed in animal models (Izumi et al, 2006). Prior treatment studies have generally focused on only particular subsets of facial emotions, a single subliminal or supraliminal condition, and a single medication as the first steps toward demonstrating the involvement of the amygdala in the treatment of MDD.

These prior studies using emotion probes have not focused on whether pre-treatment amygdala activation is a predictor of subsequent clinical response to antidepressants. Our aim was to advance our knowledge about prediction by assessing a cohort of MDD participants who were randomized to three commonly prescribed antidepressants. Drawing on the existing evidence for the effects of treatment on amygdala activation we tested the working hypotheses that (1) pretreatment amygdala activity is a general predictor of treatment response to these commonly prescribed antidepressants and (2) pre-treatment amygdala activity can differentially predict response by medication class. We also examined whether treatment-predictive amygdala activity at baseline differs between MDD participants and healthy participants, whether it changes with treatment and as a function of medication response, and whether it depends on a particular emotion or level of processing (subliminal and supraliminal). We assessed patients from the neuroimaging component of the International Study to Predict Optimized Treatment for Depression (iSPOT-D) (Williams et al, 2011; Grieve et al, 2013). Participants were randomized to one of three antidepressants in a practical clinical trial design. Amygdala activation was probed by multiple expressions of fear, anger, sadness and happiness processing in both subliminal and supraliminal conditions (Korgaonkar et al, 2013).

\section{PATIENTS AND METHODS}

\section{Participants}

Functional imaging data were drawn from 80 participants with MDD and 34 matched healthy participants who completed pre- and post-treatment sessions at the Westmead Hospital teaching hospital of the Sydney Medical School (Supplementary Figure 1). Sample size and power were determined as part of the protocol development (Grieve et al, 2013). Our power calculation was designed to test for an anticipated general effect of small effect size or higher and an anticipated differential effect with a medium effect size or higher, requiring at least 29 participants per treatment arm. See Table 1 for participant characteristics.

A complete description of the iSPOT-D design, clinical assessments, and inclusion/exclusion criteria is provided in previous papers on the study protocol (Williams et al, 2011; Grieve et al, 2013). In short, the primary diagnosis of nonpsychotic MDD was confirmed using the Mini-International Neuropsychiatric Interview (MINI) (Sheehan et al, 1998), according to DSM-IV criteria, and a score $\geqslant 16$ on the 17item Hamilton Rating Scale for Depression $\left(\mathrm{HRSD}_{17}\right)$ (Hamilton, 1960). Our $\mathrm{HRSD}_{17}$ cut-off score of 16 slightly exceeded the $\mathrm{HRSD}_{17}$ cut-off score of 14 used in $\mathrm{STAR}^{\star} \mathrm{D}$ (Trivedi et al, 2006), and is consistent with the recommended cut-offs for identifying patients with depression severity the very high end of the mild through to moderate and severe range (Zimmerman et al, 2013).

All MDD participants were either antidepressant medication naïve or, if previously prescribed an antidepressant medication, had undergone a wash-out period of at least 1 week (five half-lives). Healthy control participants were extensively screened for the absence of Axis I disorders (using the MINI) and for an $\mathrm{HRSD}_{17}$ score $\leqslant 7$, and they could not meet the DSM-IV criteria for recurrent or nonrecurrent MDD or other psychiatric disorders.

This study was conducted according to the principles of the Declaration of Helsinki 2008. After the study procedures were fully explained in accordance with the ethical guidelines of the institutional review board, participants provided written informed consent.

\section{Study Treatments}

Participants were randomized to receive escitalopram, sertraline or venlafaxine-XR using PhaseForward's validated, 
Table I Sample Characteristics for Responders/Remitters vs Non-Responders/Remitters

\begin{tabular}{|c|c|c|c|c|c|c|c|c|c|c|c|c|}
\hline \multirow[t]{2}{*}{ Characteristics } & \multirow{2}{*}{\multicolumn{2}{|c|}{ Controls $(n=34)$}} & \multirow{2}{*}{\multicolumn{2}{|c|}{ MDD $(n=80)$}} & \multicolumn{4}{|c|}{ Response } & \multicolumn{4}{|c|}{ Remission } \\
\hline & & & & & Yes $(r$ & : 48) & No $(n$ & 32) & Yes $(n$ & :37) & No $(n$ & 43) \\
\hline Gender & Mean & SD & Mean & SD & Mean & SD & Mean & SD & Mean & SD & Mean & SD \\
\hline $\begin{array}{l}\text { Clinician-rated symptom severity } \\
\left(\mathrm{HRDS}_{17} \text { score }^{\mathrm{a}}\right.\end{array}$ & - & - & 21.1 & 3.9 & 21.9 & 4.1 & 20.1 & 3.3 & 21.8 & 4.3 & 20.6 & 3.5 \\
\hline $\begin{array}{l}\text { Self-rated symptom severity } \\
\text { (QIDS-SR } 16 \text { score) }\end{array}$ & - & - & 13.8 & 3.6 & 14.2 & 3.6 & 13.0 & 3.6 & 14.1 & 4.0 & 13.5 & 3.3 \\
\hline Age of onset & - & - & 20.5 & 11.0 & 18.7 & 7.7 & 23.1 & 14.3 & 19.5 & 7.7 & 21.3 & 13.2 \\
\hline Disease duration ${ }^{b}$ & - & - & 11.8 & 11.7 & 10.5 & 10.6 & 13.8 & 13.1 & 8.3 & 6.5 & 14.8 & 14.2 \\
\hline
\end{tabular}

Abbreviations: HRSD 17, 17-item Hamilton Rating Scale for Depression; MDD, major depressive disorder; QIDS-SR 16 , 16-item quick inventory of depressive symptomatology — self-rated; $X R$, extended release.

Information for healthy controls is provided for comparison.

${ }^{a}$ Difference between responders and non-responders at $p<0.05$. Responders were on an average younger than non-responders $(p=0.006)$ and had slightly higher symptom severity as rated by the HDRSI7 ( $p=0.044)$. The sub-sample breakdown by treatment and response/non-response status was as follows: Escitalopram ( 17 responders, I 0 non-responders), Sertaline (I 6 responders, II non-responders) and Venlafaxine-XR (I 5 responders, II non-responders).

${ }^{b}$ Difference between remitters and non-remitters at $p<0.05$. Remitters were also on an average younger than non-remitters $(p=0.002)$.

Web-based Interactive Response Technology. A blocked randomization procedure was undertaken centrally (block size of 12 , across sites). Investigators/raters and participants were not blind to treatment assignment. Medications were prescribed and doses were adjusted by treating clinicians according to routine clinical practice, and following the recommended dose ranges.

Any treatment for concurrent general medical conditions were allowed and recorded. Comorbid general medical conditions were recorded under the categories (with examples) of cardiovascular (hypertension), digestive (IBS), endocrine (diabetes), hemic/lymphatic (gout), metabolic/ nutritional (high cholesterol), musculoskeletal (tendonitis), respiratory (asthma), urogenital (kidney stone), skin (ecze$\mathrm{ma}$ ), and special senses (astigmatism) disorders. Approximately $50 \%$ of the sample reported no comorbid general medical condition in these categories, $23 \%$ reported one condition and $27 \%$ one or more conditions.

Psychotropic medication was discontinued prior to randomization except for occasional (ie, $\leqslant 1$ dose/week) use of anxiolytics, sleep aids, and medications to manage antidepressant-induced side-effects (eg, nausea) as they reflect common practice. Of the total sample, $4.9 \%$ of patients were taking a concomitant psychotropic medication and these (by generic name) included the anxiolytic Alprazolam and the sedative/hypnotics, Zolpidem, Zopiclone, Eszopiclone, and Triazolam.

\section{Criteria for Response and Remission}

Study outcomes were treatment response, defined as $a \geqslant 50 \%$ decrease from the baseline $\mathrm{HRSD}_{17}$, and remission, defined as a score of $\leqslant 7$ on the $\mathrm{HRSD}_{17}$ (Williams et al, 2011). We analyzed data for participants who completed the post-treatment scanning session (Table 1), consistent with previous studies that included a post-treatment scan (Sheline et al, 2001; Fu et al, 2004).

\section{Facial Emotion Paradigms}

The emotion paradigms are summarized in Figure 1, and their viability for studying MDD has been established previously (Korgaonkar et al, 2013). Facial expression stimuli were drawn from a standardized series of facial expressions of threat-related emotions (fear and anger), loss-related emotions (sadness), and reward-related emotions (happiness), along with neutral (Gur et al, 2002), modified to be centrally positioned at eye level.

In the subliminal condition, emotional expressions were presented below the level of conscious awareness. Each face was presented briefly $(16.7 \mathrm{~ms})$, followed immediately by a neutral face perceptual mask for $150 \mathrm{~ms}$ and an interstimulus interval of $1233.3 \mathrm{~ms}$. Neutral masked faces stimuli were offset slightly by 1 degree in random directions to control for the possible detection of emotions based on perceptual features (eg, the apparent motion in the pairing of a fear face with upraised eyebrows followed by a neutral face mask compared with the pairing of an angry face with contracted eyebrows followed by a neutral face mask). Using behavioral psychophysical testing, we have shown that when faces in this paradigm are presented at $\leqslant 20 \mathrm{~ms}$, they meet signal detection criteria for being at the subliminal threshold for detection, such that individual participants cannot detect the 


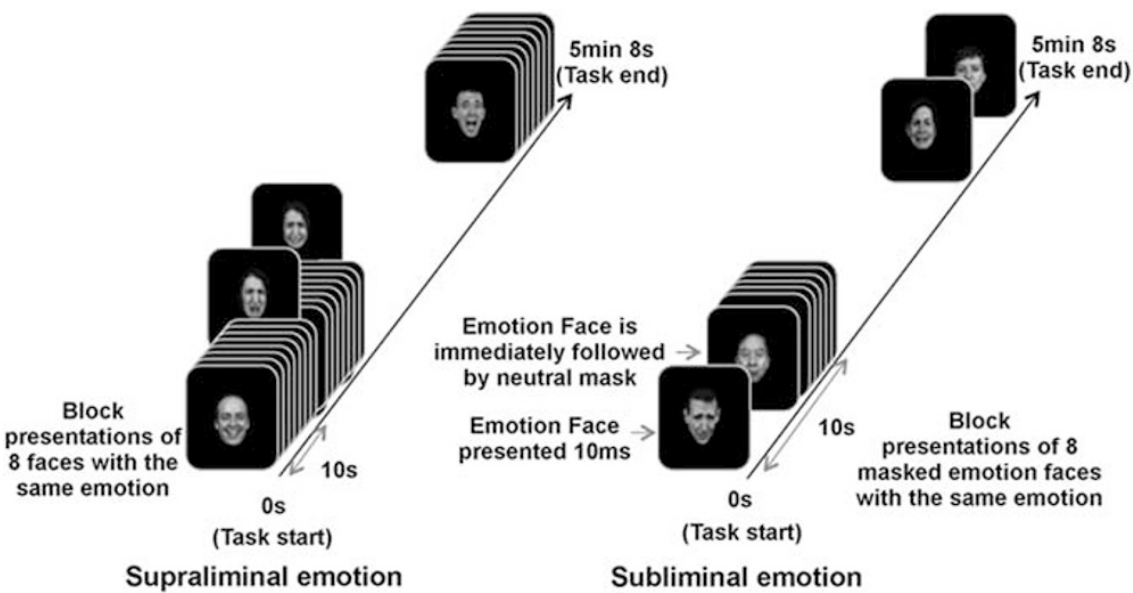

Figure I Summary of subliminal and supraliminal emotion paradigms.

presence of the face nor discriminate the facial expression (Williams et al, 2004).

In the supraliminal paradigm, we used the same emotional faces but they were presented for $500 \mathrm{~ms}$, with an interstimulus interval of $750 \mathrm{~ms}$. The stimulus duration of $500 \mathrm{~ms}$ was based on evidence that a duration of $500 \mathrm{~ms}$ is appropriate for ensuring conscious elaborative processing of the emotion stimulus. Conscious discrimination of emotion is consistently above chance (and close to 100\%) at durations $\geqslant 330 \mathrm{~ms}$ (Williams et al, 2004) and facial expressions of emotion consistently elicit a contagious effect of experiencing the emotion signaled by the stimulus at durations of $500 \mathrm{~ms}$ (Wild et al, 2001). Stimulus onset asynchrony was standardized at $1250 \mathrm{~ms}$ across both subliminal and supraliminal paradigms.

In the subliminal and supraliminal paradigms, stimuli were grouped in blocks of eight faces of the same emotion, with each emotion block repeated five times in pseudorandom order. A total of 240 stimuli were presented within each paradigm. No specific behavioral responses were required during scanning because of the inclusion of subliminal presentations and our aim to isolate activation elicited by emotion stimuli independent of behavioral task demands. A large meta-analysis of 385 studies has shown passive processing is associated with a higher probability of activation than an active task (Costafreda et al, 2008). Because stimuli were presented via goggles, stimuli filled participant's field of view. We created a context for participants to continuously view the faces by instructing them that they would be asked post-scan questions about these faces.

\section{Image Acquisition}

MRIs were acquired using a 3.0 T GE Signa HDx scanner (GE Healthcare, Milwaukee, Wisconsin). Acquisition was performed using an 8-channel head coil and an echo planar imaging (EPI) sequence with the following parameters: $\mathrm{TR}=2500 \mathrm{~ms}$, $\mathrm{TE}=27.5 \mathrm{~ms}$, matrix $=64 \times 64, \mathrm{FOV}=24 \mathrm{~cm}$, flip angle $=90^{\circ}$. Forty contiguous axial/oblique slices with a slice thickness of $3.5 \mathrm{~mm}$ were acquired to cover the whole brain in each volume. For each paradigm, 120 volumes were collected (one for every two face stimuli) with a total scan time of $5 \mathrm{~min}$ and $8 \mathrm{~s}$. Three dummy scans were acquired at the start of every acquisition.

Structural MRI 3D T1-weighted images were acquired in the sagittal plane using a 3D spoiled gradient echo (SPGR) sequence $\quad\left(\mathrm{TR}=8.3 \mathrm{~ms} ; \mathrm{TE}=3.2 \mathrm{~ms} ; \quad\right.$ Flip Angle $=11^{\circ}$; $\mathrm{TI}=500 \mathrm{~ms} ; \mathrm{NEX}=1 ; \mathrm{ASSET}=1.5$; Frequency direction: $\mathrm{S} / \mathrm{I}$ ). A total of 180 contiguous $1 \mathrm{~mm}$ slices were acquired, covering the whole brain with a $256 \times 256$ matrix with an in-plane resolution of $1 \times 1 \mathrm{~mm}$, resulting in $1 \mathrm{~mm}^{3}$ isotropic voxels. The 3D SPGR sequence was collected for use in normalization of the fMRI data to standard space.

\section{Processing of Functional Imaging Data}

The fMRI data were preprocessed using the SPM8 software (www.fil.ion.ucl.ac.uk/spm). The details of the preprocessing methodology have been described previously (Korgaonkar et al, 2013). In brief, motion correction using realignment and unwarping, global signal removal and smoothing using an $8 \mathrm{~mm}$ Gaussian kernel was performed for both fMRI tasks. For normalization to stereotactic Montreal Neurological Institute (MNI) space, the T1-weighted data were normalized to standard space using the fMRI of the Brain (FMRIB) nonlinear registration tool and the FMRI EPI data was coregistered to the T1 data using the FMRIB linear registration tool. Normalization warps from these two steps were stored for use in functional to standard space transformations.

The left and right amygdala regions-of-interest (ROIs) were defined by an anatomical mask using the Automated Anatomical Labeling (AAL) atlas with maarsbar-aal-0.2 (Brett et al, 2002; Tzourio-Mazoyer et al, 2002). The ROIs were therefore the same for all participants. We then controlled for multiple comparisons by using a voxel wise family-wise error correction $\left(p_{\mathrm{FWE}}=0.05\right)$. Locations of peak activation were defined using MNI coordinates.

A hemodynamic response convolved boxcar function was used to model the Blood Oxygenation Level-Dependent (BOLD) response for each of the emotion blocks. In the firstlevel fixed-effect analysis, images were derived for the contrast of emotion $v s$ neutral. Individual contrast images were normalized to standard space using the normalization warps estimated in the preprocessing steps above. The 
standard space contrast maps were then entered for all second-level random effects analyses.

The statistical analysis was designed in a step-wise manner to address study aims and their clinical relevance as follows:

\section{Analysis of General Prediction}

Step 1: To test whether pre-treatment amygdala activation relates generally to post-treatment antidepressant outcomes, we used a general linear model (GLM) in SPM8 with treatment outcome (binary classification as responder/remitter or non-responder/remitter at 8 weeks post treatment) as the between-participants factor. Covariates were severity of depressive symptoms (assessed by the $\mathrm{HRSD}_{17}$ ) and age, given that responder/remitter and non-responder/remitter subgroups differed slightly on these variables (Table 1).

A priori contrasts tested whether responders/remitters and non-responders/non-remitters differed on each emotion grouping, with all three treatment arms taken together

Step 2: For significant prediction effects identified in the first step, we extracted each participant's parameter estimates ( $\beta$ weights) of amygdala activation, an index of BOLD signal change for emotion minus neutral. To undertake our planned contrasts $\beta$ weights were extracted from the specific voxels that defined the cluster of significant activation. These $\beta$ weight data were analyzed using IBM SPSS software, version 21 . We first compared responders and non-responders with healthy controls using the GLM with a repeated measure for threat (fear and anger) and $t$-tests for sad and happy. We then used the GLM with the time (pre $v s$ post treatment) as a within-participants factor, and response/remission status as the between-participants factor, to assess change from pre- to post-treatment within the same amygdala clusters.

\section{Analysis of Differential Prediction}

Step 1: To test whether activation is differentially affected by the type of antidepressant, we used the GLM in SPM8 with a second between-participants factor for antidepressant type (with levels for the SSRIs escitalopram and sertraline, and the serotonin-norepinephrine reuptake inhibitor (SNRI) venlafaxine-XR). Covariates were again depression severity $\left(\mathrm{HRSD}_{17}\right)$ and age. We tested for the interaction between response/remission status and antidepressant treatment type. These interaction analyses were undertaken for the amygdala defined as it was for general prediction analyses with the same correction for multiple comparisons.

Step 2: For the significant differential prediction effects that were identified in the first step, we extracted $\beta$ weights from the amygdala ROI and used the GLM and $t$-tests to compare responders and non-responders within each medication type to healthy controls. We then used the GLM to test whether the interactions between response/remission status and type of antidepressant that were revealed at Step 1 changed or persisted over time, in a three-way interaction with time (ie, any difference in amygdala activation in the time period from pre- to post-treatment).

\section{Correlations with Symptoms}

When a significant difference was found between responders and non-responders, correlations were run to examine the degree of association between amygdala activation and $\mathrm{HRSD}_{17}$-rated symptoms within each response status subgroup. When significant pre-post treatment changes were identified, we used correlation analysis to examine the degree of association between change in amygdala activation and change in $\mathrm{HRSD}_{17}$-rated symptoms.

\section{Effect Sizes}

To aid in the interpretation of the findings, we computed effect sizes using Cohen's $d$ or partial $\eta^{2}$ converted to Cohen's $d$ equivalents.

\section{Classification Sensitivity and Specificity}

To aid in interpretation of the findings in regard to potential clinical applicability, we undertook secondary analyses using a discriminant analysis classifier method to evaluate the classification accuracy for predictors of general and differential prediction. In these classifiers, we used the leave one out' method to cross-validate the classification model. We also assessed the contribution of amygdala activation over and above the contribution of pre-treatment symptom severity, and age.

\section{RESULTS}

\section{Response and Remission Rates}

The rates of response and remission are presented in Table 1. When participants were stratified by treatment outcome (responder/non-responder and remitter/non-remitter), the two groups differed significantly in depressive symptom severity and in age. These variables were taken into account as covariates in subsequent imaging analyses.

From herein, we focus on responders $v s$ non-responders since it was for this comparison that significant amygdala activation effects were revealed. No significant effects in amygdala activations were observed in relation to remission.

\section{General Prediction of Response by Amygdala Activation to Subliminal Happy and Threat Expressions}

We focus on subliminal amygdala activation as a general predictor of response because no significant general prediction effects were observed for the supraliminal condition. The negative results for the supraliminal condition are unlikely to be due to the paradigm manipulation given the robust amygdala activation for healthy controls in this condition (for details see Supplementary Results).

In the Step 1 voxel-wise analyses with FWE correction, responders showed lower activation of the left and right amygdala for subliminal processing of faces that signal potential reward (ie, happy expressions) compared with nonresponders (Table 2; Figure $2 \mathrm{a}$ and $\mathrm{b}$ ). This difference in responders $v s$ non-responders had a strong effect size. Responders also showed lower activation of the left amygdala for the subliminal processing of faces that signal threat (ie, fear and anger expressions) with a strong effect size (Table 2; Figure 2c). 
Table 2 Summary of Step Wise Results: Step (I) a priori Voxel Wise Analyses to Test for General and Differential Prediction of Responders (R) vs Non-Responders (NR), and Step (2) Follow-Up Analyses of the Prediction Effects Revealed at Step I to Assess for Pre-Treatment Differences to the Normative Control Baseline and for Pre-Post Treatment Changes

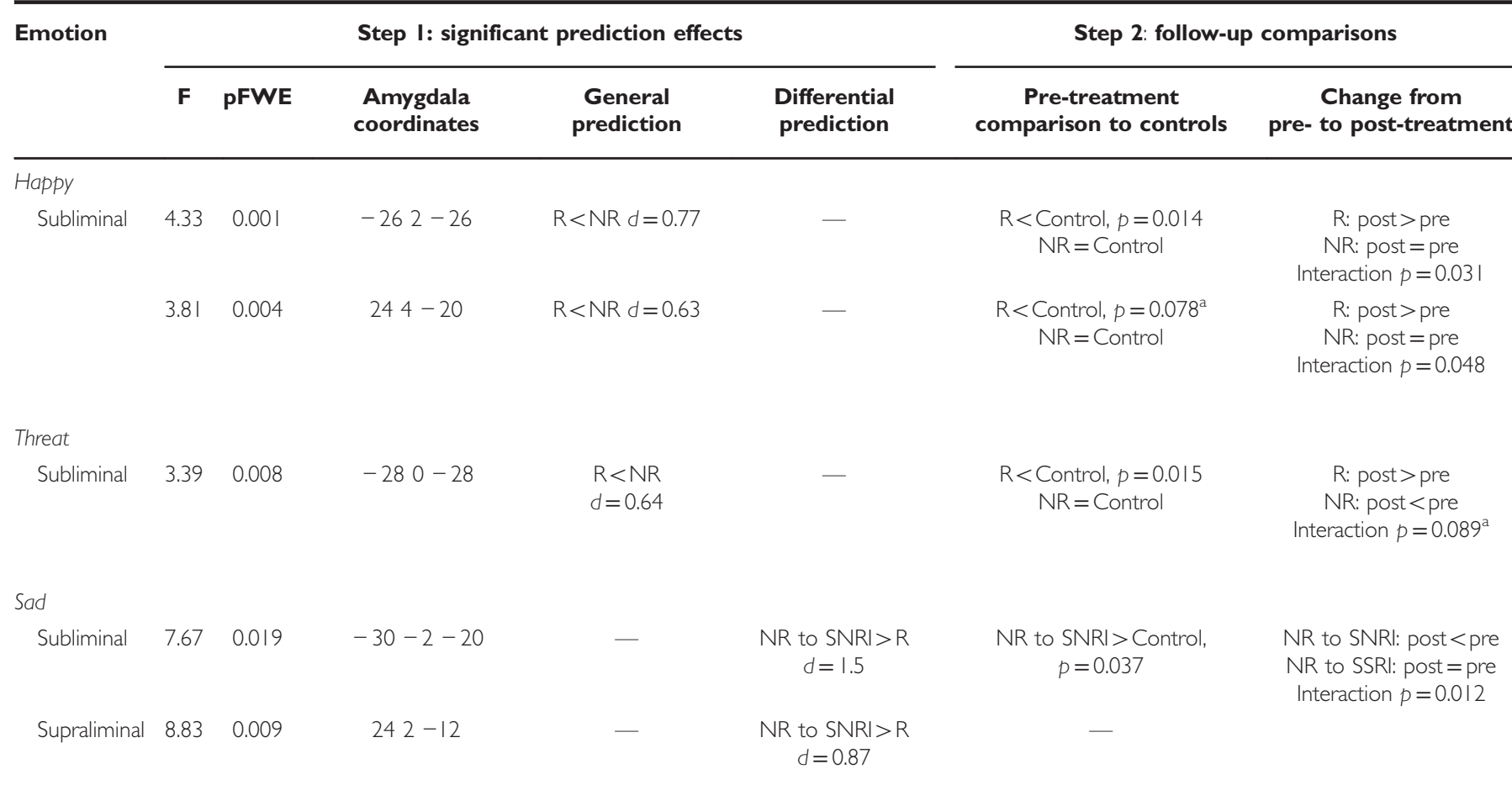

Abbreviations: MNI, Montreal Neurological Institute; NR, non-responder, pFWE, p-value with family-wise error correction; R, responder; SNRI, serotoninnorepinephrine reuptake inhibitor, SSRI, selective serotonin reuptake inhibitor.

At Step 2 ' $=$ ' is used to signify a non-significant difference. $d=$ Cohen's $d$ indicator of effect size.

a'Trend level effect.

At Step 2, when we looked at treatment-outcome-predictive amygdala activation extracted from the clusters of significant effect, $t$-tests showed that this activation was reduced for subliminal happy in responders compared with healthy controls at pre-treatment baseline, with the difference being significant for the left amygdala and at trend level for the right amygdala (Table 2; Figure 2a and $b$ ). The GLM revealed an interaction between response status and pre-post treatment change in amygdala activation for subliminal happy. This interaction was due to responders showing an increase in bilateral amygdala activation for subliminal happy, with the increase being in the direction of post-treatment 'normalization' (Table 2; Figure $2 \mathrm{a}$ and $\mathrm{b}$ ). At post-treatment, these responders no longer differed from controls (Table 2; Figure 2a and b). In responders, the degree of pre-post treatment increase in left amygdala activation was significantly and positively associated with the degree of improvement in symptoms $(r=0.24, p=0.035)$. Non-responders showed a tendency toward reduced activation for subliminal happy post-treatment, but this reduction was not significant, and non-responders did not differ significantly from controls at either pre-treatment baseline or post-treatment (Table 2; Figure $2 a$ and $b$ ).

Similarly, the GLM showed that treatment-predictive left amygdala activation was also reduced for subliminal threat in responders compared with controls (Table 2; Figure 2c). Responders showed a trend interaction for pre-post treatment change that was due to an increase in amygdala activation for threat, but non-responders showed a relative decrease (Table 2; Figure 2c). In responders, the posttreatment increase in left amygdala activity was positively associated with the degree of symptom improvement $(r=0.30, p=0.037)$. There were no correlations between activation and dose within each of the treatment arms.

\section{Differential Prediction of Response by Amygdala Activation to Sad Expressions}

In a voxel-wise analysis at Step 1, we found an interaction between outcome and type of medication for left amygdala activation during subliminal processing of sad faces (Table 2; Figure 3). This interaction was driven by a specific effect for non-responders in response to a specific type of medication, the SNRI venlafaxine-XR (in contrast to the general predictive effects above, which were driven by responders). The differential effect of subliminal sad-elicited amygdala activation for non-responders $v s$ responders to venlafaxineXR had a very strong effect size (Table 2). Non-responders to venlafaxine-XR showed comparative hyper-activation of the left amygdala to subliminal sad expressions, which progressed post treatment into comparative hypo-activation to these expressions (Table 2; Figure 3). By contrast, responders to SSRIs showed a trend towards amygdala hypo-activation to sad $(p=0.06$, Figure 3$)$, consistent with the general predictive effects for happy and threat.

At Step 2, $t$-tests showed apparent hyper-activation to subliminal sad expressions in SNRI non-responders 


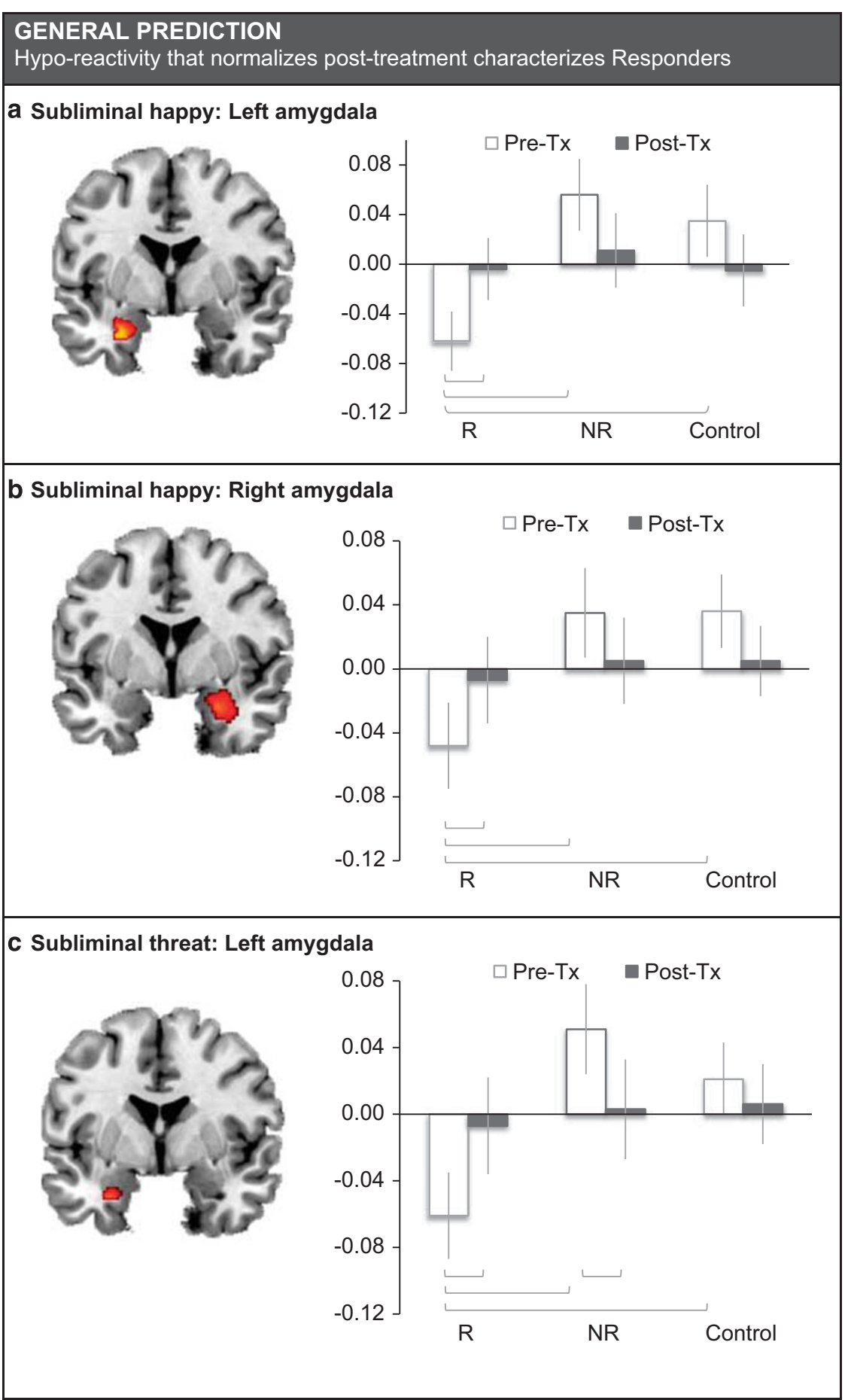

Figure 2 Summary of results for amygdala activation as a general predictor of response outcome for subliminal happy and threat faces. Gray lines indicate significance between group differences. Mean activation data presented in bar charts include age as a covariate. NR, non-responder; R, responder.

compared with controls at the pre-treatment baseline, and hypo-activation compared with controls at the posttreatment phase (Table 2; Figure 3). GLM modeling of the activation data revealed a three-way interaction between prepost treatment changes, response status and antidepressant type due to a post-treatment 'abnormal hypo-activation' (ie, opposite to normalization) of the amygdala in SNRI nonresponders (Table 2; Figure 3). In correlation analyses, any change in amygdala activity for these non-responders was independent of the degree of symptom change, and there were no correlations between activation and dose.

There was a corresponding interaction for right amygdala activation during supraliminal processing of sad expressions, also due to hyper-activity in non-responders compared with responders (Table 2). In this case, however, hyper-activity was not present in non-responders to the extent that it 


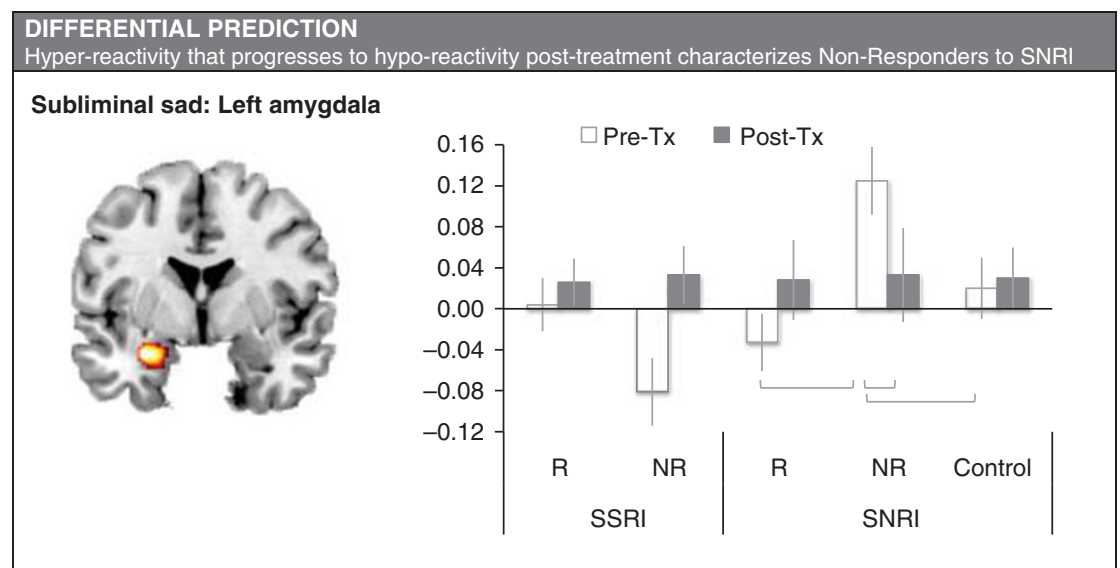

Figure 3 Summary of results for amygdala activation as a differential predictor of response outcome for subliminal happy and threat faces. Gray lines indicate significance between group differences. Mean activation data presented in bar charts include age as a covariate. NR, non-responder; R, responder; SNRI, serotonin-norepinephrine reuptake inhibitor; SSRI, selective serotonin reuptake inhibitor.

differed significantly from controls or changed significantly post treatment.

\section{Classification Sensitivity and Specificity}

In light of the present findings, we conducted a secondary analysis of the sensitivity and specificity of amygdala activity in classifying responders and non-responders to the antidepressant medications in this study. We focused on the subliminal condition for which both general and differential predictive effects were robust. The goal of this exploratory analysis was a step towards identifying a functional imaging metric for helping guide treatment decisions.

General prediction. A summed composite of bilateral amygdala hypo-activation for subliminal reward and left amygdala activation for subliminal threat was computed as the simplest composite given the common direction of effect in responders $v s$ non-responders for these emotions. In the discriminant classification model, amygdala activation and age (but not $\mathrm{HRSD}_{17}$-rated pre-treatment symptom severity) contributed significantly to prediction of response status $\left(\lambda=0.74 ; \chi^{2}=23.66, \mathrm{df}=2, p<0.0001\right)$. This model classified responders $v s$ non-responders with an overall accuracy of $75 \%$. The range of accuracy for classification based on individual metrics of amygdala activation for the left and right amygdala for subliminal happy and the left amygdala activation for subliminal threat was $73-78 \%$, indicating that the metric of summed amygdala activation was appropriate. Sensitivity for prediction of response was $77 \%$ and specificity for non-response was $72 \%$. The cross-validation accuracy was also $75 \%$, suggesting that the classification model is generalizable. Accuracy with covariates alone was $61 \%$. Thus, the inclusion of amygdala reactivity to happy and threat improved predictive accuracy by $14 \%$.

Differential prediction. Amygdala reactivity to subliminal sad expressions and the covariate of age (but not $\mathrm{HRSD}_{17}$-rated pre-treatment symptom severity) accurately contributed to a significant classification of response $v s$ non-response to the SNRI $\left(\lambda=0.82 ; \chi^{2}=15.03, \mathrm{df}=2, p=0.002\right)$. The overall accuracy of this model was $81 \%$ with a sensitivity of $87 \%$ and specificity of $73 \%$. The cross-validation accuracy of $77 \%$ suggested that this classification is generalizable. Amygdala activation improved differential predictive accuracy by $20 \%$ over and above covariates alone. By contrast, amygdala activation for subliminal sad expressions only contributed $7 \%$ additional predictive accuracy for response vs nonresponse to SSRIs.

\section{DISCUSSION}

In this study, we report on new evidence for the role of emotion-elicited amygdala activation in the general prediction of antidepressant treatment outcomes, and the differential moderation of response to specific types of commonly prescribed antidepressants. The findings suggest that the patient's pre-treatment level of amygdala activation to subliminally processed facial expressions that signal reward and threat is generally predictive of which patients will respond to antidepressants. On the other hand, pre-treatment amygdala activation to facial expressions that signal sadness was specifically and differentially predictive of which participants failed to respond to a specific antidepressant, the SNRI venlafaxine-XR. Amygdala predictive activation was abnormal at the pre-treatment baseline for participants with MDD compared with controls. Post treatment, amygdala activation for reward and threat changed in the direction of normalization for responders in general, and sad activation changed in the direction of abnormal progressive hypo-activity for non-responders to venlafaxine-XR.

The way that amygdala circuitry reacts to different signals of emotion may provide a biomarker to indicate which individual patients have the neural capacity to respond to antidepressants as a general predictive effect, and which are unlikely to respond to SNRIs as a specific predictive effect. Expressions that signal reward and threat may engage circuitry that is involved in the response to antidepressants of multiple classes. The receptor-level pharmacological actions of medication may help restore a hypo-functioning amygdala to a more normal level of function. Expressions of both fear and happiness have been associated with neural 
activation of the amygdala that is in response to salient changes in the eyes (Meletti et al, 2012). In this context, patients with MDD who have a loss of neural capacity for processing salient signals (be it threatening or rewarding, but not mood-congruent) may benefit from the general effects that antidepressants have regarding the restoration of this capacity. On the other hand, patients who show an initial predisposing hyper-reactivity to mood-congruent signals of sadness, followed by abnormal hypo-activation, may have a non-remitting course of MDD that not only fails to normalize with treatment, especially an SNRI, but might even be exacerbated by it. Consistent with these possibilities, in responders the restoration of the amygdala from initial pre-treatment hypo-activity to post-treatment normal levels of activation was correlated with a corresponding improvement in symptoms. This profile in responders suggests that the normalization of amygdala activity goes hand in hand with the normalization of symptoms. By contrast, hyperactivity in non-responders to the SNRI progressed in the direction opposite to that of normalization (ie, exacerbation) independent of symptoms.

The most robust amygdala prediction, both general and differential, was for subliminally elicited activation, which suggests that the automatic processing of salient emotional signals (in the absence of any conscious reflection acting on these signals) may be the more direct way to probe the circuitry involved in antidepressant response. Our observation that results were most apparent for the subliminal condition accords with evidence across studies of healthy subjects that there is a greater likelihood of amygdala activation to threat stimuli when there are no overt task demands (Wild et al, 2001). This suggestion is consistent with previous evidence that amygdala activation for subliminal sadness in MDD changes following treatment (Victor et al, 2010; Arnone et al, 2012).

A new observation in the present study is that responders were defined pre-treatment by hypo-activation of the amygdala for subliminal processing of happy and threat relative to controls, while non-responders were defined by hyper-activation. A number of fMRI studies have reported amygdala hyper-reactivity in response to negative emotional faces in MDD overall relative to controls under both supraliminal (Peluso et al, 2009; Surguladze et al, 2005; Fu et al, 2004; Zhong et al, 2011) and subliminal (Sheline et al, 2001; Victor et al, 2010; Stuhrmann et al, 2012; Suslow et al, 2010) (for meta-analysis see the study by Hamilton et al (2012); for review see the studies by Browning et al (2010); Jaworska et al (2014)). These studies have typically reported on medicated patients, who by virtue of still meeting criteria for the disorder while on medication are likely non-responders. In our study, we focused on a treatment seeking sample from patients who were unmedicated at the time of their first scan. We found that hyper-reactivity of the amygdala pre-treatment (in unmedicated subjects) is present in a specific subset of MDD subjects who were subsequently found to be non-responders to an SNRI. Based on these findings, we propose that medication non-responsive patients may in particular drive prior observations of amygdalar hyper-activation in depression. In other words, by anchoring our analyses around treatment response our findings lend a new perspective on understanding clinical heterogeneity within MDD. Interestingly, amygdalar hypo-reactivity has been observed previously in fMRI studies of both adults (Lawrence et al, 2004) and of unmedicated children with MDD (Thomas et al, 2001) for fear faces. Thus, there is clearly a need for better understanding sources of heterogeneity within the disorder, for which the perspective of treatment response prediction may be particularly valuable, along with other related factors such as comorbidity and phase of illness.

It is also possible that paradigm variations drive the direction of effect in responders $v s$ non-responders to antidepressants, and that the direction is less important than the fact that pattern of brain activation differentiates response status per se. Meta-analyses of emotion-elicited amygdala hyper-reactivity have focused on the weight of evidence from more explicit tasks rather than on subliminal stimuli. Extending on this point, we might consider the present findings in the context of results for non-emotion tasks that require effortful cognition. Using a cognitive task to probe effortful inhibition, increased (rather than decreased) activation of the amygdala and regions to which it projects has been found to predict antidepressant response (Langenecker et al, 2007). In a separate study of the iSPOT-D sample, we have also observed that increased activation of the DLPFC engaged by a response inhibition task is predictive of response (Gyurak et al, 2015). It is possible therefore that, in the same patients, those individuals who are likely to benefit from an antidepressant show a combination of hypo-activation during automatic emotion processing and hyper-activation during effortful processing depending on the nature of the emotional/cognitive task they are engaged in.

Another possibility is that the direction of amygdala activation (hypo- or hyper-activation) as a predictor of antidepressant response is influenced by the baseline resting state in depressed patients. In a study using positron emission tomography to probe baseline glucose metabolism, improvement in major depressive symptoms was found to correlate with lower pre-treatment metabolism in the amygdala (Saxena et al, 2003). To tease out this issue, future fMRI studies might examine amygdala activation probed by emotion stimuli and amygdala activation during rest in the same depressed patients assessed pre and post treatment. We also need future studies that extend the investigation of both emotion-probed and resting amygdala activation to multiple treatment modalities. In an fMRI study using a paradigm designed to probe sustained emotion processing, amygdala hyper-activation was found to predict response to cognitive behavior therapy (Siegle et al, 2006). To our knowledge, amygdala activation has not been investigated as a general or differential predictor of treatment response to pharmacological and non-pharmacological treatments assessed using the same paradigms in the same study.

Limitations of the present study need to be addressed in future research. Due to the practical trial design, the study was necessarily limited to antidepressants that are in common use in each of the participating countries. It would be important to verify the differential predictive role of the amygdala with additional antidepressants that have distinct mechanisms of action, including serotonin-norepinephrinedopamine reuptake inhibitors. The analysis of clinical subphenotypes was also beyond the scope of the present study. However, in light of evidence that amygdala reactivity also 
has a role in response to antidepressant response to both SSRIs and SNRIs in anxiety disorder (Phan et al, 2013; Whalen et al, 2008), it would be important to determine whether the general prediction of response and differential prediction of non-response via amygdala activation is also indicative of distinct clinical entities that involve anxiety and other distinguishing characteristics. Focusing on subphenotypes defined by specific levels of MDD severity would also help determine the specificity of the present findings. Further investigation of sub-phenotypes also has the potential to reveal additional regions with prognostic value. For example, in small well characterized groups, higher activity in the anterior cingulate during emotion processing has been found to predict improvement following antidepressant treatment (for review and meta-analysis see the study by Fu et al, (2013)).

The present results also require replication and assessment of generalizability in an independent sample.

In conclusion, this study provides new evidence that functional imaging biomarkers of amygdala activity may ultimately help tailor treatment selection according to which patients are generally likely to respond to SSRIs and which patients are specifically unlikely to respond to SNRIs.

\section{FUNDING AND DISCLOSURE}

LMW has received fees as a consultant for Brain Resource and did hold stock in Brain Resource. AE has received research funding from Brain Resource for iSPOT-D. AWFH has received consultancy fees from Janssen Australia and Lundbeck Australia. He has received payments for educational sessions run for Janssen Australia and the Lundbeck Institute. He has recently been an investigator on industry-sponsored trials by Hoffman-La Roche, JanssenCilag Australia, and Brain Resource Ltd. SMG has received fees as a consultant for Brain Resource. The remaining authors declare no conflict of interest.

\section{ACKNOWLEDGMENTS}

LMW takes responsibility for the integrity of the data and the accuracy of the data analysis and was the Academic PI for iSPOT-D from 2008-2013. Dr Claire V.A. Day was the Global Trial Coordinator for iSPOT-D. Brain Resource was involved in the design of the study, managed the central coordination of the study, and provided the executive structure for a publication committee to manage the preparation of manuscripts. Brain Resource was not involved in the acquisition, analysis, or interpretation of the data. We gratefully acknowledge the contributions of the co-investigators and at the Sydney site where imaging data were acquired, and the Westmead Hospital MRI personnel at the Sydney site (Lavier Gomes, FRACR; Sheryl Foster). We gratefully acknowledge the editorial support of Jon Kilner (Pittsburgh, PA, USA). Clinical trials registration details are as follows: Trial registration: International Study to Predict Optimized Treatment in Depression (iSPOT-D); registry name: ClinicalTrials.gov; URL: http://www.clinicaltrials.gov/ct2/show/ NCT00693849?term=iSPOT-D\&rank=1; Registration number: NCT00693849. iSPOT-D is sponsored by the Brain Resource Company Operations.

\section{REFERENCES}

Almeida JRC, Versace A, Hassel S, Kupfer DJ, Phillips ML (2010). Elevated amygdala activity to sad facial expressions: a state marker of bipolar but not unipolar depression. Biol Psychiatry 67: 414-421.

Arnone D, McKie S, Elliott R, Thomas EJ, Downey D, Juhasz G et al (2012). Increased amygdala responses to sad but not fearful faces in major depression: relation to mood state and pharmacological treatment. Am J Psychiatry 169: 841-850.

Brett M, Anton J, Valabregue R, Poline J (2002). Region of interest analysis using an SPM toolbox. International Conference on Functional Mapping of the Human Brain. 8th edn; 2-6 June 2002; Sendai, Japan.

Browning M, Holmes EA, Harmer CJ (2010). The modification of attentional bias to emotional information: A review of the techniques, mechanisms, and relevance to emotional disorders. Cogn Affect Behav Neurosci 10: 8-20.

Costafreda SG, Brammer MJ, David AS, Fu CH (2008). Predictors of amygdala activation during the processing of emotional stimuli: a meta-analysis of 385 PET and fMRI studies. Brain Res Rev 58: 57-70.

Fu CH, Steiner H, Costafreda SG (2013). Predictive neural biomarkers of clinical response in depression: a meta-analysis of functional and structural neuroimaging studies of pharmacological and psychological therapies. Neurobiol Dis 52: 75-83.

Fu CHY, Williams SCR, Cleare AJ, Brammer MJ, Walsh ND, Kim J et al (2004). Attenuation of the neural response to sad faces in major depression by antidepressant treatment: a prospective, event-related functional magnetic resonance imaging study. Arch Gen Psychiatry 61: 877-889.

Godlewska BR, Norbury R, Selvaraj S, Cowen PJ, Harmer CJ (2012). Short-term SSRI treatment normalises amygdala hyperactivity in depressed patients. Psychol Med 25: 1-9.

Grieve SM, Korgaonkar MS, Etkin A, Harris A, Koslow SH, Wisniewski S et al (2013). Brain imaging predictors and the international study to predict optimized treatment for depression: study protocol for a randomized controlled trial. Trials 14: 224.

Gur RC, Sara R, Hagendoorn M, Marom O, Hughett P, Macy L et al (2002). A method for obtaining 3-dimensional facial expressions and its standardization for use in neurocognitive studies. J Neurosci Methods 115: 137-143.

Gyurak A, Williams L.M, Patenaude B, Korgaonkar MS, Grieve SM, Etkin A (2015). Fronto-parietal activation during response inhibition predicts remission to antidepressants in patients with major depression. Biol Psychiatry (http://dx.doi.org/10.1016/ j.biopsych.2015.02.037).

Hamilton JP, Etkin A, Furman DJ, Lemus MG, Johnson RF, Gotlib IH (2012). Functional neuroimaging of major depressive disorder: a meta-analysis and new integration of base line activation and neural response data. Am J Psychiatry 169: 693-703.

Hamilton M (1960). A rating scale for depression. J Neurol Neurosurg Psychiatry 23: 56-61.

Harmer CJ, Mackay CE, Reid CB, Cowen PJ, Goodwin GM (2006). Antidepressant drug treatment modifies the neural processing of nonconscious threat cues. Biol Psychiatry 59: 816-820.

Izumi T, Inoue T, Kitaichi Y, Nakagawa S, Koyama T (2006). Target brain sites of the anxiolytic effect of citalopram, a selective serotonin reuptake inhibitor. Eur J Pharmacol 534: 129-132.

Jaworska N, Yang XR, Knott V, Macqueen G (2014). A review of fM42.RI studies during visual emotive processing in major depressive disorder. World J Biol Psychiatry in the press.

Korgaonkar MS, Grieve SM, Etkin A, Koslow SH, Williams LM (2013). Using standardized fMRI protocols to identify patterns of prefrontal circuit dysregulation that are common and specific to cognitive and emotional tasks in major depressive disorder: first wave results from the iSPOT-D study. Neuropsychopharmacology 38: $863-871$. 
Langenecker SA, Kennedy SE, Guidotti LM, Briceno EM, Own LS, Hooven $\mathrm{T}$ et al (2007). Frontal and limbic activation during inhibitory control predicts treatment response in major depressive disorder. Biol Psychiatry 62: 1272-1280.

Lawrence NS, Williams AM, Surguladze S, Giampietro V, Brammer MJ, Andrew C et al (2004). Subcortical and ventral prefrontal cortical neural responses to facial expressions distinguish patients with bipolar disorder and major depression. Biol Psychiatry 55: 578-587.

Meletti S, Cantalupo G, Benuzzi F, Mai R, Tassi L, Gasparini E et al (2012). Fear and happiness in the eyes: an intra-cerebral eventrelated potential study from the human amygdala. Neuropsychologia 50: 44-54.

Murphy SE, Norbury R, O’Sullivan U, Cowen PJ, Harmer CJ (2009). Effect of a single dose of citalopram on amygdala response to emotional faces. Br J Psychiatry 194: 535-540.

Peluso MA, Glahn DC, Matsuo K, Monkul ES, Najt P, Zamarripa F et al (2009). Amygdala hyperactivation in untreated depressed individuals. Psychiatry Res 173: 158-161.

Phan KL, Coccaro EF, Angstadt M, Kreger KJ, Mayberg HS, Liberzon I et al (2013). Corticolimbic brain reactivity to social signals of threat before and after sertraline treatment in generalized social phobia. Biol Psychiatry 73: 329-336.

Ruhé HG, Booij J, Veltman DJ, Michel MC, Schene AH (2012). Successful pharmacologic treatment of major depressive disorder attenuates amygdala activation to negative facial expressions: a functional magnetic resonance imaging study. J Clin Psychiatry 73: 451-459.

Rush AJ, Wisniewski SR, Warden D, Luther JF, Davis LL, Fava M et al (2008). Selecting among second-step antidepressant medication monotherapies: predictive value of clinical, demographic, or first-step treatment features. Arch Gen Psychiatry 65: 870-880.

Saxena S, Brody AL, Ho ML, Zohrabi N, Maidment KM, Baxter LR (2003). Differential brain metabolic predictors of response to paroxetine in obsessive-compulsive disorder versus major depression. Am J Psychiatry 160: 522-532.

Sheehan DV, Lecrubier Y, Sheehan KH, Amorim P, Janavs J, Weiller E et al (1998). The Mini-International Neuropsychiatric Interview (M.I.N.I.): The development and validation of a structured diagnostic psychiatric interview for DSM-IV and ICD-10. J Clin Psychiatry 59(Suppl. 20): 22-33.

Sheline YI, Barch DM, Donnelly JM, Ollinger JM, Snyder AZ, Mintun MA (2001). Increased amygdala response to masked emotional faces in depressed subjects resolves with antidepressant treatment: an fMRI study. Biol Psychiatry 50: 651-658.

Siegle GJ, Carter CS, Thase ME (2006). Use of fMRI to predict recovery from unipolar depression with cognitive behavior therapy. Am J Psychiatry 163: 735-738.

Stuhrmann A, Dohm K, Kugel H, Zwanzger P, Redlich R, Grotegerd D et al (2012). Mood-congruent amygdala responses to subliminally presented facial expressions in major depression: associations with anhedonia. J Psychiatry Neurosci 38: 249-258.

Stuhrmann A, Suslow T, Dannlowski U (2011). Facial emotion processing in major depression: a systematic review of neuroimaging findings. Biol Mood Anxiety Disord 1: 10.

Surguladze S, Brammer MJ, Keedwell P, Giampietro V, Young AW, Travis MJ et al (2005). A differential pattern of neural response toward sad versus happy facial expressions in major depressive disorder. Biol Psychiatry 57: 201-209.

Suslow T, Konrad C, Kugel H, Rumstadt D, Zwitserlood P, Schoning $S$ et al (2010). Automatic mood-congruent amygdala responses to masked facial expressions in major depression. Biol Psychiatry 67: 155-160.
Thomas KM, Drevets WC, Dahl RE, Ryan ND, Birmaher B, Eccard $\mathrm{CH}$ et al (2001). Amygdala response to fearful faces in anxious and depressed children. Arch Gen Psychiatry 58: 1057-1063.

Townsend JD, Eberhart NK, Bookheimer SY, Eisenberger NI, Foland-Ross LC, Cook IA et al (2010). fMRI activation in amygdala and orbitofrontal cortex in unmedicated subjects with major depressive disorder. Psychiatry Res 183: 209-217.

Trivedi MH, Rush AJ, Wisniewski SR, Nierenberg AA, Warden D, Ritz L et al (2006). Evaluation of outcomes with citalopram for depression using measurement-based care in $\mathrm{STAR}^{\star} \mathrm{D}$ : implications for clinical practice. Am J Psychiatry 163: 28-40.

Tzourio-Mazoyer N, Landeau B, Papathanassiou D, Crivello F, Etard O, Delcroix N et al (2002). Automated anatomical labeling of activations in SPM using a macroscopic anatomical parcellation of the MNI MRI single-subject brain. Neuroimage 15: 273-289.

Victor TA, Furey ML, Fromm SJ, Ohman A, Drevets WC (2010). Relationship between amygdala responses to masked faces and mood state and treatment in major depressive disorder. Arch Gen Psychiatry 67: 1128-1138.

Vuilleumier P, Pourtois G (2007). Distributed and interactive brain mechanisms during emotion face perception: evidence from functional neuroimaging. Neuropsychologia 45: 174-194.

Whalen PJ, Johnstone T, Somerville LH, Nitschke JB, Polis S, Alexander AL et al (2008). A functional magnetic resonance imaging predictor of treatment response to venlafaxine in generalized anxiety disorder. Biol Psychiatry 63: 858-863.

Whalen PJ, Shin LM, Somerville LH, McLean AA, Kim H (2002). Functional neuroimaging studies of the amygdala in depression. Semin Clin Neuropsychiatry 7: 234-242.

Wild B, Erb M, Bartels M (2001). Are emotions contagious? Evoked emotions while viewing emotionally expressive faces: quality, quantity, time course and gender differences. Psychiatry Res 102: 109-124.

Williams LM, Das P, Liddell BJ, Kemp AH, Rennie CJ, Gordon E (2006). Mode of functional connectivity in amygdala pathways dissociates level of awareness for signals of fear. J Neurosci 26: 9264-9271.

Williams LM, Gordon E (2007). Dynamic organization of the emotional brain: Responsivity, stability, and instability. Neuroscientist 13: 349-370.

Williams LM, Liddell BJ, Rathjen J, Brown KJ, Shevrin H, Gray JA et al (2004). Mapping the time course of nonconscious and conscious perception of fear: An integration of central and peripheral measures. Hum Brain Mapp 21: 64-74.

Williams LM, Rush AJ, Koslow SH, Wisniewski SR, Cooper NJ, Nemeroff $\mathrm{CB}$ et al (2011). International study to predict optimized treatment for depression (iSPOT-D), a randomized clinical trial: rationale and protocol. Trials 12: 4.

World Health Organization (2012). Mental Health: Mental Health Atlas 2011 Available from http://www.who.int/mental_health/ publications/mental_health_atlas_2011/en/index.html.

Yang TT, Simmons AN, Matthews SC, Tapert SF, Frank GK, Max JE et al (2010). Adolescents with major depression demonstrate increased amygdala activation. J Am Acad Child Adolesc Psychiatry 49: 42-51.

Zhong M, Wang X, Xiao J, Yi J, Zhu X, Liao J et al (2011). Amygdala hyperactivation and prefrontal hypoactivation in subjects with cognitive vulnerability to depression. Biol Psychiatry 88: $233-242$.

Zimmerman M, Martinez JH, Young D, Chelminski I, Dalrymple K (2013). Severity classification on the Hamilton Depression Rating Scale. J Affect Disord 150: 384-388.

Supplementary Information accompanies the paper on the Neuropsychopharmacology website (http://www.nature.com/npp) 